\title{
Occupy London und die besetzte Friern Barnet Library
}

\begin{abstract}
Johannes Diesing
Der Gegenstand des Beitrages ist die besetzte Friern Barnet Library in Nord-London. Dieser steht für eine ,Politik des Politischen', die sich gegen die Privatisierungsmaßnahmen der regierenden liberal-konservativen Koalition richtet. In diesem Kampf um die Bibliothek wurden Aussagen der programmatischen Vision der Regierung von einer Allianz aus Aktivist_innen und Bürger_innen polemisch gegen die Politik gewendet, deren Umsetzung diese Vision ideologisch legitimieren sollte. Es werden zunächst die Rahmenbedingungen der Besetzung beschrieben, danach wird detailliert auf das Geschehen im Herbst 2012 und Winter 2012/13 eingegangen. Es wird erklärt, warum die Auseinandersetzung um die Bibliothek für das Verständnis gegenwärtiger sozialer Kämpfe in der ,postpolitischen Stadt' hilfreich ist. Zum Begriff der Postpolitik bzw. der Postdemokratie haben sowohl Colin Crouch als auch Jacques Rancière Theorien vorgelegt. Der Aufsatz wird den theoretischen Schwerpunkt auf Rancière legen, Crouchs Überlegungen jedoch ebenfalls mit einbeziehen.
\end{abstract}

Ersteinreichung: 1. April 2013; Veröffentlichung online: 7. Dezember 2013

An english abstract can be found at the end of the document.

Von der U-Bahn Station Arnos Grove führt die Friern Barnet Road durch ein Gebiet von zweistöckigen Wohnhäusern, vorbei am Gelände des ehemaligen Colney Hatch Lunatic Asylum, einer alten Nervenklinik, zur Friern Barnet Libary, die in einem dem Tudorstil nachempfundenen Backsteingebäude beherbergt ist. An das Gelände schließt eine Siedlung neuer Einfamilienhäuser an. Und obwohl selbst der konservative Evening Standard im Oktober 2012 auch diesem Stadtteil im Norden Londons eine Wohnungskrise bescheinigte (Prynn 2012), scheint die Bevölkerung hier David Camerons Vision der ,ownership society ${ }^{\natural}$ zu entsprechen, die der Premier in einer Rede unmittelbar nach den riots im August 2011 als ein Ideal beschworen hatte (Cameron 2011). Der in diesem Aufsatz dargestellte Kampf um die besetzte Friern Barnet Libary steht indes exemplarisch für eine ,Politik des Politischen', die sich gegen die Kürzungs- und Kommerzialisierungsmaßnahmen der liberal-konservativen Regierung richtet. In diesem Kampf um die Bibliothek wurden Aussagen der programmatischen Vision der Regierung von einer Allianz aus Aktivist_ innen und Bürger_innen polemisch gegen die Politik gewendet, deren Umsetzung diese Vision ideologisch legitimieren sollte.

Bis zum 5. April 2012 war die Friern Barnet Libary eine öffentliche Gemeindebibliothek. Dann kam das Ende. Der Bestand der Bücher sollte 
in eine andere Bibliothek im sehr weitläufigen Stadtbezirk Barnet überführt werden. Eine lokale Kampagne hatte bis zuletzt vergeblich versucht, die öffentliche Bibliothek zu retten. Nach ihrer Schließung stand das Gebäude zum Verkauf. Einen vom Gemeinderat versprochenen Ersatz gab es nicht. Im September 2012 besetzten dann Aktivist_innen von Occupy London das Gebäude und eröffneten erneut eine Bibliothek.

Dieser Aufsatz wird zunächst die Rahmenbedingungen der Besetzung beschreiben und dann detailliert auf das lokale Geschehen im Herbst 2012 und im Winter 2012/13 eingehen,[1] Der Schwerpunkt liegt auf den Aktivitäten von Occupy London und auf den besonderen Schwierigkeiten und Herausforderungen, die sich für die Bewegung nach der gewaltsamen Räumung ihrer Camps im Innenstadtbereich ergaben. Vor allem wird jedoch erläutert, warum die Auseinandersetzung um die Friern-Barnet-Bibliothek besonders aufschlussreich für das Verständnis gegenwärtiger sozialer Kämpfe in der postpolitischen Stadt ist. Zum Begriff der Postpolitik bzw. der Postdemokratie haben sowohl Colin Crouch als auch Jacques Rancière Theorien vorgelegt. Der Grund für die Entscheidung, den theoretischen Schwerpunkt hier auf Rancière zu legen, resultiert aus der Überzeugung, dass dessen Theorie besser geeignet ist, das ,Neue' am politischen Handeln der Occupy-Bewegung zu erfassen. Gleichwohl entwickelte Crouch sein Konzept der Postpolitik vor dem Hintergrund der Geschichte des britischen politischen Systems, weshalb auch seine Beiträge in diesen Aufsatz mit einfließen. Zum Abschluss werden dann die Schwierigkeiten der Theorie von Jacques Rancière u. a. in Bezug auf die Dimensionen Raum und Zeit diskutiert.

\section{Der Hintergrund der Besetzung der Bibliothek}

Um die Besetzung der Bibliothek zu verstehen, ist es notwendig, auf drei Faktoren und Entwicklungen einzugehen, die den Hintergrund für die Ereignisse in Barnet abgeben. Der erste Faktor ist das „One Barnet Transformation Programme“ des konservativ geführten Gemeinderats, der - um die zentralstaatlichen Kürzungsvorgaben zu erfüllen - auf eine umfassende Kommerzialisierung öffentlicher Dienstleistungen setzte. Das Programm sah vor, den Auftrag zur Übernahme von 70 Prozent der kommunalen Dienstleistungen für die Dauer von zehn Jahren an das Unternehmen Capita zu vergeben. Ziel des One Barnet Programme ist eine umfassende Umstrukturierung der öffentlichen Dienstleistungen im Stadtbezirk (Barnet Council 2010). Es wurde vom 2010 gewählten Gemeinderat, in dem die Konservativen mit 39 Sitzen eine Mehrheit haben, aufgelegt. Nach dessen Plänen sollten 70 Prozent der kommunalen Dienstleistungen ausgeschrieben und an eine private Firma vergeben werden. In diesem Verfahren setzte sich ein auf die Übernahme von Verwaltungs- und Behördendienstleistungen spezialisiertes Unternehmen, die Capita Group, mit einem Angebot durch, welches der Gemeinde über 320 Millionen Pfund einbringen sollte. Der daraufhin geschlossene Vertrag sollte eine Laufzeit von zehn Jahren haben (20132023) und den Angaben des Barnet Council zufolge in dieser Zeit 125 Millionen Pfund an Steuergeldern einsparen (vgl. Blunden 2012). 
Nach einer sehr kurzfristigen Bekanntmachung der Schließung der Friern Barnet Libary, die am 5. April 2012 stattfand, kam es zu einer Protestkundgebung vor dem Gebäude und einer ersten Besetzung des Hauses durch Aktivist_innen der lokalen Kampagne „Save the Friern Barnet Libary" für die Dauer von fünf Stunden. Der Protest hatte allerdings keine aufschiebende Wirkung. Der Bibliotheksbetrieb wurde ersatzlos eingestellt.

Zum besseren Verständnis des Kontextes der späteren Besetzung durch Aktivist_innen von Occupy London ist es darüber hinaus notwendig, die Herausforderungen zu betrachten, mit denen die Bewegung im Fortgang des Jahres 2012 konfrontiert war. Spätestens nach der Räumung der beiden großen Camps auf dem Vorplatz der St.-Pauls-Kathedrale im Februar 2012 und auf dem Finsbury Square im Juni 2012 stellte sich für die OccupyBewegung die Frage, wie sie politisch weitermachen wollte. Die Bewegung, zu deren wichtigsten Praxen das buchstäbliche Besetzthalten von öffentlichem Raum gehörte, stand nun ohne einen festen Ort dar. Angesichts des näher rückenden Jahrestages der Platzbesetzung vor der St.-PaulsKathedrale, dem eine hohe symbolische Bedeutung zukam, stellte sich im Spätsommer immer dringlicher die Frage: Was tun?

Hinzu kam eine weitere Entwicklung, die sich im frühen Herbst zuspitzte. Im September trat in Großbritannien ein Gesetz in Kraft, das bis zu diesem Zeitpunkt legale Besetzungen von leerstehendem Wohnraum zum Zwecke des dauerhaften Bewohnens unter Strafe stellte. Section 144 des „Sentencing and Punishment of Offenders Act 2012" definiert nun als Straftat, wenn eine Person in ein Gebäude mit der expliziten Absicht eindringt, längere Zeit dort zu bleiben (Ministry of Justice 2012). Dieses neue Gesetz hatte weitreichende Folgen, weil es das Konfliktfeld von Häuserbesetzungen in Großbritannien völlig neu regelte. Spätestens nachdem im September ein 21-Jähriger wegen der Besetzung einer Wohnung zu zwölf Monaten Gefängnis verurteilt worden war, war die Londoner Hausbesetzerszene alarmiert. In den folgenden Wochen und Monaten kam es zu einer Reihe von Neubesetzungen von leerstehenden Gebäuden in kommunalem oder privatem Besitz, die nicht als Wohnraum klassifiziert sind und damit nicht unter das neue Gesetz fielen. Eines davon war die ehemalige Friern Barnet Libary. Da sich das Gebäude nicht zur Umwandlung in Wohnraum eignete, nahmen die Besetzer_innen Kontakt zu Occupy-London auf. Die OccupyAktivist_innen entwickelten eine Strategie und verkündeten öffentlich die Besetzung der Räumlichkeiten und die Wiedereröffnung der Bibliothek (vgl. Shamsuddin 2012).

Die Hinwendung von Londoner Aktivist_innen aus der Occupy-Bewegung weg von Massenaktionen hin zu lokalen Aktionen und Besetzungen war kein Einzelfall. Ähnliche Tendenzen ließen sich auch in anderen Ländern nach der polizeilichen Räumung der innerstädtischen Zeltlager beobachten. In Spanien etwa entstand mit der „Plataforma de Afectados por la Hiptoteca" ein organisatorischer Rahmen, um die Proteste der Bewegung 15M gegen eine ganz bestimmte Auswirkung der Krise weiterzuführen: Zwangsräumungen. Zum einen versuchte man, Zwangsräumungen zu verhindern, zum anderen leerstehenden Wohnraum zu besetzen. Auch in der US-amerikanischen Occupy-Bewegung vollzog sich nach der Räumung der 
innerstädtischen Camps mit „Occupy our Homes“ eine Hinwendung zum Thema Zwangsräumungen, mit wechselhaftem Erfolg.

\section{Der große Ausverkauf - Privatisierung als Kommerzialisierung}

Die Kürzungen und Privatisierungen der öffentlichen Dienstleistungen, in deren Folge die Bibliothek in Barnet geschlossen wurde, sind fester Bestandteil der Agenda der liberal-konservativen Regierung unter David Cameron. In deren Zentrum steht die Annahme, dass die private Hand immer bessere Resultate erzielen kann als der Staat.[2] Dem One Barnet Programme zufolge soll die Auslagerung der Dienstleistungen für die Bürger_innen eine Qualitätssteigerung mit sich bringen (The Barnet Council 2010). An solchen Privatisierungsvorhaben kritisiert hingegen Colin Crouch (2008: 129) mit Bezug auf den Arbeitsrechtsexperten Mark Freedland, dass durch die Auslagerung von öffentlichen Diensten an private Firmen eine trianguläre Beziehung von Regierung, Bürger_innen und privaten Dienstleistern entstehe, die zu einer Verantwortungsdiffusion führe.

Dramatisch sind solche Probleme aufgrund des Umfangs der Reformen. Im Wahlkampf des Jahres 2010, den die Konservativen gewannen, hatte der Begriff der ,big society“ eine wesentliche Rolle gespielt. Unmittelbar nach ihrem Amtsantritt begann die konservativ-liberale Regierung mit ihrem Vorhaben, den ,großen Staat' abzubauen. Die umfassende Privatisierung öffentlicher Dienstleistungen sollte durch eine aktivierte Bürgergesellschaft aufgefangen werden.

„Durch den Versuch, Alternativen zum Staat zu finden, damit er in dem Maße ,schrumpfen' kann, wie es die Neoliberalen fordern, steigen die Ansprüche an karitative Organisationen und ehrenamtliche Helfer. [...] Dies [...] zeigt sich [...] vor allem am ,Big Society'Programm der rechtsliberalen britischen Koalition, die alle möglichen gesellschaftlichen Gruppen aufruft, mögliche Aufgaben, bei denen sie den öffentlichen Dienst einst unterstützten, nunmehr komplett zu übernehmen.” (Crouch 2011: 232)

Im Fall des One Barnet Programme ging es um die Privatisierung von 70 Prozent der kommunalen Dienstleistungen (Blunden 2012). Ob den Anwohner_innen diese Dimension klar gewesen ist, lässt sich nur schwer sagen. Eine Occupy-Aktivistin äußerte die Einschätzung, dass die Menschen im Stadtteil vor allem ihre Bibliothek sehen, aber nur wenige die größeren Zusammenhänge der Privatisierung erkennen, sodass dies quasi hinter ihrem Rücken durchgeführt werden kann. Der Erfolg des Engagements der OccupyAktivist_innen hing somit auch davon ab, inwieweit es ihnen gelingen sollte, den größeren Kontext der kommunalen Kürzungspolitik aufzuzeigen.

Nach der öffentlichen Bekanntgabe der Besetzung bemühten sich die Besetzer_innen um eine Vernetzung mit den lokalen Aktivist_innen, die sich bereits im Frühjahr für den Erhalt der Bibliothek engagiert hatten. Gemeinsam mit diesen und den Anwohner_innen gelang es ihnen, den Bibliotheksbetrieb wieder aufzunehmen. Eine offensive Öffentlichkeitsarbeit führte zu einer regen Berichterstattung. Zu den Alltagsaufgaben gehörte die 
Begrüßung und Begleitung von Bibliotheksbesucher_innen, die Annahme von Bücherspenden, das Einsortieren der Bücher, die Reinigung des Gebäudes und der umliegenden Flächen, die Vorstellung des Projekts in der Nachbarschaft, die Planung und Durchführung von Veranstaltungen sowie von Beratungsgesprächen, die Reparatur des kaputten Dachs und die Instandhaltung der Computer und Elektronik. Die Aktivist_innen richteten Facebook-Seiten und Twitter-Accounts ein und schrieben Berichte, um die Bewegungsöffentlichkeit auf dem Laufenden zu halten.

Ingesamt haben die Anwohner_innen über 8.000 Bücher für die Bibliothek gespendet. Bei den meisten Titeln handelte es sich um Unterhaltungsliteratur. Explizit politische Schriften finden sich vor allem in Form zahlreicher Flyer, Zeitungen und Plakate der verschiedenen NGOs, lokalen Initiativen oder Bündnisse gegen Sozialkürzungen. Die Bücher der Bibliothek waren also nicht durch ihre Themen ein Vehikel zur Agitation der Nachbarschaft. Die Politisierung der Anwohner_innen fand vielmehr durch die Partizipation an den lokalen Kämpfen zum Erhalt der Bibliothek statt. Wichtig für die weitere Politisierung waren der Transfer praktischer Bewegungserfahrungen sowie vielfältige Prozesse der Vernetzung und Solidarisierung. Menschen kamen miteinander in Kontakt, die sich ansonsten nie kennen gelernt hätten. Die besetzte Bibliothek stiftete Raum für neue Begegnungen. Die Nachbarschaft konnte sich ein eigenes Bild von der Occupy-Bewegung machen, ohne den Filter der Medien.

Nach Auskunft einer der Aktivist_innen entspricht dies der aktuellen strategischen Ausrichtung von Occupy London. Die Bewegung konzentriert sich auf die Unterstützung von lokalen Initiativen und im zweiten Schritt auf eine bessere Vernetzung untereinander, sodass im Falle einer drohenden Räumung von besetzten Gebäuden und Projekten auch andere Initiativen mit Solidaritätsaktionen einspringen können. Wichtig ist ihnen dabei, dass die lokalen Gruppen und Initiativen in der Nachbarschaft gut verankert sind und sich quer zu klassischen politischen Zuordnungen positionieren, was z.B. die Gefahr einer Kooptierung vonseiten der Parteien mindert. Solche Initiativen sollen den Anwohner_innen die Chance bieten, mehr zu tun, als nur im konventionellen Sinne - etwa durch die Teilnahme an Wahlen - an Politik zu partizipieren. Die Occupy-Bewegung setzt damit ihren Anspruch um, möglichst vielen Menschen den Zugang zu anderen Formen des politischen Handelns, abseits der parlamentarischen Demokratie, zu eröffnen. Aus ihrer Sicht verschaffte die Beteiligung an der Besetzung und Wiedereröffnung der Bibliothek vielen Anwohner_innen ganz neue Erfahrungen, z.B. die, dass es keinen Widerspruch zwischen den politischen Zielen und den eingesetzten Mitteln geben muss. Der weit verbreiteten Entfremdung - angesichts einer Regierungspolitik, die gleichzeitig Kürzungen durchpeitscht und sich einer ,big-society'-Rhetorik bedient - wollten die Besetzer_innen etwas anders entgegensetzen, nämlich die Einsicht, dass das eigene Engagement sinnvoll und erfolgreich sein kann.

Die Bibliothek und die damit verbundenen Aktivitäten bildeten vordergründig das neue Zentrum von Occupy London. Sie eröffneten ein neues ,Gelegenheitsfenster für eine kollektive Praxis als Bewegung und gewährten einer nicht unbeträchtlichen Zahl von Aktivist_innen eine Unterkunft. Allerdings war die Lage eher prekär, was die Frage aufwarf, warum die 
geschrumpfte und in der Presse bereits für tot erklärte Occupy-Bewegung annahm, in Barnet erfolgreich zugunsten der lokalen Bevölkerung intervenieren zu können. Die Londoner Occupy-Bewegung hatte zu ihren besten Zeiten, als sie zentrale Plätze in der City of London besetzt hielt, Tausende Menschen umfasst. Auch nach den Räumungen dieser Plätze war die Bewegung immer noch ein wichtiger Informationsknotenpunkt und -multiplikator. Einerseits hatte sie eigene Kanäle der GraswurzelBerichterstattung geschaffen, die immer noch genutzt werden, darunter eine eigene Homepage, auf der Aktivist_innen Diskussionen führen sowie Artikel und Beiträge einstellen können, das Occupy News Network, eine an das Prinzip von Indymedia angelehnte Internetseite mit Fokus auf die Aufstände und Protestbewegungen von 2011, und die Protestzeitung The Occupied Times of London. Des Weiteren benutzte die Bewegung sehr erfolgreich soziale Netzwerke zum Verbreiten von Nachrichten und auch von eigenen Inhalten. Besondere Bedeutung hatte der Livestreamdienst www. bambuser.com, mit dem viele der Protestaktionen, aber auch gewöhnliche Aktivist_innen-Treffen live ins Internet übertragen wurden. Unter den Occupy-Aktivist_innen waren auch immer Ansprechpartner_innen für die Medien zu finden. Die daraus resultierende große Bekanntheit der Bewegung wirkte im Herbst 2012 immer noch nach. Occupy funktionierte weiterhin als eine Art buzzword, dementsprechend hatte auch die Schlagzeile „Occupy-AktivistInnen besetzen Bibliothek” immer noch einen Nachrichtenwert. Selbst internationale Journalist_innen interessierten sich für die Besetzung der Friern Barnet Library in Nord-London.

\section{Politik, Polizei und Occupy - Jacques Rancières und die Praxis demokratischer Kämpfe}

Die Schließung der Gemeindebibliothek im Rahmen des One Barnet Programme stellt ein Paradebeispiel für eine postpolitische bzw. postdemokratische Politik dar. Dabei ist es wichtig, sich die gewandelten Lebensrealitäten zu vergegenwärtigen, mit denen viele der Bewohner_innen angesichts der anhaltenden Hegemonie des Neoliberalismus zu kämpfen haben. Dass es ausgerechnet in einem politisch konservativen Bezirk mit einer starken gehobenen Mittelschicht gelang, einen klaren Dissens zu neoliberalen Strategien wie der Privatisierung von öffentlicher Dienstleistungen zu vertreten, liefert über den konkreten Fall hinaus Hinweise für eine emanzipatorische Perspektive in der postdemokratischen Stadt. Neben den konkreten Handlungs- und Aktionsformen erscheint insbesondere eine ästhetische Komponente des Kampfes interessant, der im Folgenden mit Hilfe des französischen Post-Marxisten Jacques Rancière nachgegangen werden soll.

Zunächst ist es jedoch notwendig, kurz die für das besondere Demokratieverständnis des Autors zentrale Differenz zwischen den Begriffen der Politik und der Polizei zu erläutern. Mit Polizei bezeichnet Rancière weniger die Gesamtheit der Repressionsbehörden und auch nicht den Staat im engeren Sinne. Die Polizei ist für ihn viel weiter gefasst eine Ordnung des Sichtbaren und des Sagbaren (Rancière 2002: 41). Politik ist demgegenüber die Unterbrechung dieser Ordnung des Sichtbaren und Sagbaren. „Der 
Unterschied wird zwischen einem jeweils bestehenden System selbstregulierender Prozesse und einem sie durchbrechenden Ereignis gezogen, das sie in Frage stellt" (Bedorf 2010: 25). Demokratie ist daher nach Rancière auch keine Herrschaftsform oder gesellschaftliche Lebensweise.

„Sie ist die Einsetzung der Politik selbst, das System der Formen der Subjektivierungen, durch welche jede Verteilung der Körper nach Funktionen, die ihrer Natur entspricht, und nach den Plätzen die ihren Funktionen entsprechen, in Frage gestellt, auf ihre Kontingenz verwiesen wird.“ (Rancière 2002: 111)

Demokratie ist also das, was den normalen, durch Gesetze geregelten Ablauf in den Institutionen unterbricht, die üblicherweise in der Politikwissenschaft als das politische System bezeichnet werden, bei Rancière aber mit dem Namen Polizei versehen ist. Die Polizei ist eine Ordnung des Sichtbaren und des Sagbaren, die dafür zuständig ist, dass den Körpern, die sie ordnet durch ihre Namen ein bestimmter Platz und eine bestimmte Aufgabe zugewiesen ist. (Ranciere 2002: 41) Der Polizei liegt Rancière zufolge eine Aufteilung des Sinnlichen zugrunde, die in einer Aufzählung der vorhandenen Teile und einer Beschreibung ihrer Ordnung besteht. „Die Aufteilung des Sinnlichen legt dadurch fest, was Teil jener Wirklichkeit ist, die sich als ,natürliche' Ordnung präsentiert, und was keine Berechtigung innerhalb dieser Erfahrungswelt hat." (Mörtenböck/ Mooshammer 2012: 116). Politik unterbricht diese Ordnung, indem sie aufzeigt, wer aus dieser ausgeschlossen ist. „Gerade der so oft geäußerte Vorwurf an die Occupy-Bewegung, keine bedeutsamen Forderungen zu haben oder aber Unmögliches zu fordern, macht deutlich, wie eng dieser Rahmen ist, in dem eine Forderung als real gilt" (ebd.).

Wie findet nun eine solche Unterbrechung der Polizei statt? Politik, schreibt Rancière, ist eine Sache der Subjekte oder vielmehr der Subjektivierungsweisen.

„Unter Subjektivierungsweisen [Hervorheb.i.O.] wird man eine Reihe von Handlungen verstehen, die eine Instanz und eine Fähigkeit zur Aussage erzeugen, die nicht im gegebenen Erfahrungsfeld identifizierbar waren, deren Identifizierung also mit einer Neuordnung des Erfahrungsfeldes einhergeht." (Rancière 2002: 47)

Der Begriff der Subjektivierung meint hier also das In-Erscheinung-treten einer Äußerung, die ein Ausgeschlossensein eines Teils der Gesellschaft offenlegt und damit einhergehend einen Dissens artikuliert. Es geht darum, die Art und Weise, wie die Ordnung der Dinge bzw. das politische System funktionieren, durch das Auftreten einer Unterbrechung, die die Ungerechtigkeit eines Ausschlusses thematisiert, in einem neuen Licht erscheinen zu lassen. Denn die politische Tätigkeit ist für Rancière jene, „die einen Körper von dem Ort entfernt, der ihm zugeordnet war oder die die Bestimmung eines Ortes ändert; sie lässt sehen, was keinen Ort hatte, gesehen zu werden, lässt eine Rede hören, die nur als Lärm gehört wurde“ (Rancière 2002: 41). Tatsächlich wurde an der Occupy-Bewegung nicht nur eine fehlende inhaltliche Klarheit kritisiert. Der Bürgermeister von London beschimpfte die Aktivist_innen im November 2011 zudem als 
„hemp-smoking, fornicating hippies“ (Kingsley 2011). Dies ist sicherlich ein drastisches Beispiel, es zeigt allerdings beispielhaft das Unvernehmen, das zwischen der Sicht des konservativen Bürgermeisters auf die Politik und den Äußerungen der protestierenden Aktivist_innen besteht. Die Intervention oder der Bruch im Sinne Rancières liegt in der Offenlegung dieses Umstandes. Der Protest zeigt auf, wer keinen legitimen Platz im System hat - wer zum Anteil gehört, der keinen Anteil hat - und fordert eine Neuverteilung der Plätze ein.

Der Blick auf die Politik der Occupy-Bewegung darf dabei nicht auf explizit getätigte Aussagen und erhobene Forderungen beschränkt werden. Der theoretische Zugang Rancières ermöglicht es, die gesamte Praxis als eine ästhetische Strategie der Intervention in das bestehende politische System zu verstehen, die offenlegt, dass es Menschen gibt, die aus diesem ausgeschlossen sind. Peter Mörtenböck und Helge Mooshammer schreiben in diesem Sinne, dass die „mit den Occupy-Bewegungen im Zusammenhang stehenden Ästhetiken [sich] nicht lediglich in ihrer zweckgerichteten Form als visuelle Repräsentation des Protestes sehen [lassen], sondern als global verteilte Intervention in den Bereich der Erfahrbarkeit, als Demonstration der Pluralität wirklicher Welten“(Mörtenböck/Mooshammer 2012: 116). Wird die Occupy-Bewegung aus diesem Blickwinkel heraus interpretiert, lässt sich mit den Worten von Rancière behaupten, die Aktivist_innen haben im Jahre 2011 mit ihrem Slogan „We are the 99\%“ eine Trennung des Demos von sich selbst vorgenommen und einen,Anteil der Anteillosen' eingerichtet, der auf eine Neuzählung und Neuverteilung der Anteile der Gesellschaft pochte. Eine solche Artikulation der Ungerechtigkeit der neoliberalen Regierungspolitik findet sich auch in der lokalen Auseinandersetzung um die Friern Barnet Library im Herbst 2012. Der Kampf der lokalen Protestinitiative wird durch die Allianz mit der Occupy-Bewegung zu mehr als nur einer institutionellen und juristischen Auseinandersetzung um die Rechtmäßigkeit eines lokalen Verwaltungsaktes.

Warum die Bewohner_innen des eher wohlhabenden Stadtteils Barnet zu einem ,Anteil der Anteillosen“ gehören sollten, ist erklärungsbedürftig. Denn als Mitglieder eines Gemeinwesens haben sie vermittelt über politische Wahlen Anteil an der Regierung, die sie somit über sich selbst ausüben. Die Polizei - in Rancières Sinne - besteht in diesem Falle darin, den Bürger_innen ihre Rolle zuzuweisen, wählen zu gehen und sich regieren zu lassen. Nicht vorgesehen ist allerdings, dass sich die Menschen des Stadtteils anschließend gegen die Politik des gewählten Gemeinderats wenden, die ja im Sinne der Bürger_innen ist, weil durch sie die Bürger_innen ihren Willen vertreten. Die Politik der Besetzung durchbricht diesen tautologischen Schluss. Der einzurichtende ,Anteil der Anteillosen“ wird dabei in Rancières Theorie nicht als eine empirisch aufzufindende gesellschaftliche Gruppe definiert (Rancière 2008: 28). Der ,Anteil der Anteillosen“ muss vielmehr zusätzlich zu allen empirischen Zählungen sowie Auf- und Einteilungen der Bevölkerungen konstituiert werden. Die Bewohner_innen von Barnet sind mehrheitlich auch nicht anteillos in dem Sinne, dass sie nicht stimmberechtigt wären oder aufgrund ihrer Armut von gesellschaftlicher Teilhabe ausgeschlossen wären. Obwohl es für die Mittelschicht in dem Stadtteil zunehmend schwieriger wird, eigene Häuser zu erwerben und 
damit dem Ideal der ,homeownership society zu entsprechen, ist gerade die gesellschaftliche Teilhabe durch Konsum nicht verstellt. Verstellt ist im Diskurs der Regierungspolitik die Behauptung der Möglichkeit einer gesellschaftlichen Alternative.

Dies ist der Punkt, an dem die subversive Politik von Occupy London ansetzen konnte. Aktivist_innen und Bürger_innen richteten sich als ,Anteil der Anteillosen' ein, indem sie sich einen Raum aneigneten, der es ihnen ermöglichte, sich als engagierte Bürger_innen zu konstituieren. Sie griffen in die polizeiliche Ordnung ein, die sagte, dass es keine Bibliotheken mehr braucht, weil billige Bücher auch im Supermarkt zu haben sind. Sie richteten sich als ,Anteil der Anteillosen' ein, indem sie aufzeigten, dass eine für die Nutzer_innen kostenlose Gemeindebibliothek mit geschultem und öffentlich finanziertem Personal keinen Platz mehr in der Welt des One Barnet Programme und der ,big society ${ }^{6}$ hat.

\section{Die postdemokratische, big society'}

Der Kampf um die Gemeindebibliothek in Friern Barnet muss vor dem spezifischen Hintergrund einer starken postdemokratischen Tendenz betrachtet werden. Nach der Ablösung von New Labour vertrat die liberal-konservative Koalition in Großbritannien eine Regierungspolitik, die noch stärker als die der Vorgänger im Amt postdemokratisch ist. Unter den Begriffen Postpolitik und Postdemokratie werden Entwicklungen in den westlichen liberalen Repräsentativdemokratien theoretisiert, die sich grundlegend auf den Kern dessen auswirken, was unter dem Begriff der Demokratie in der Regel verstanden wird. Mit dem Ende der Blockkonfrontation traten die Demokratien in den westlichen Industrieländern in eine Zeit ein, in der ein Abschied von den großen Ideologien gefeiert wurde. Doch diese Wandlungen betrafen nicht nur ideologische [3] Auseinandersetzungen, auch in den politischen Systemen selbst kam es zu weitreichenden Veränderungen. Dazu zählt der sukzessive Bedeutungsverlust von politischen Debatten und inhaltlichen Auseinandersetzungen gegenüber sogenannten Expert_innengremien, eine umfassende Privatisierung und Kommerzialisierung des öffentlichen Sektors, eine Umdeutung der Rolle der Bürger_innen zu Kund_innen und damit einhergehend der permanente Einsatz von Methoden der Markt- und Meinungsforschung, um die aufkommenden Wünsche, Stimmungen und Meinungen der Bevölkerung frühzeitig wahrnehmen, zukünftige Entwicklungen antizipieren und mit entsprechende Angeboten darauf reagieren zu können. Im Ergebnis gerät beim Fortschreiten dieser Entwicklung ein wesentliches Element der Demokratie immer mehr in den Hintergrund: nämlich die Meinungsbildung im Zuge des Streits von verschiedenen miteinander konkurrierenden Ideen und das aktive politische Handeln der Bürger_innen. Auch weil die meisten etablierten politischen Parteien diese Entwicklung unterstützt haben, gibt es für die Bürger_innen auf der institutionellen Ebene immer weniger substanzielle Möglichkeiten der legitimen und konventionellen Partizipation an Entscheidungen und damit auch weniger Möglichkeiten, sich bestimmten Maßnahmen zu widersetzen. Hinzu kommt die Tendenz der sukzessiven Ersetzung dieser politischen Teilhabe durch die Ausweitung des privaten 
Konsums. Da wo wie auf einem Markt jede möglicherweise aufkommende Nachfrage unmittelbar durch ein Angebot befriedigt werden kann, muss nichts mehr erstritten werden. [4] Der Begriff der Postdemokratie dient Rancière in diesem Sinne dazu, das Paradox zu bezeichnen, dass unter dem Namen der Demokratie die konsensuelle Praxis der Auslöschung der Formen demokratischen Handelns geltend gemacht wird.

„Die Post-Demokratie ist die Regierungspraxis und die begriffliche Legitimierung einer Demokratie nach dem Demos [Hervorheb.i.O.], einer Demokratie, die die Erscheinung, die Verrechnung und den Streit des Volkes liquidiert hat, reduzierbar also auf das alleinige Spiel der staatlichen Dispositive und der Bündelung von Energien und gesellschaftlichen Interessen." (Rancière 2002: 111)

An die Stelle der wiederkehrenden Artikulationen von Dissens rückt die Antizipation und Anerkennung von aufkommenden Interessen in der Bevölkerung. Die Politik der liberal-konservativen Koalition in Großbritannien ist deswegen postdemokratisch, weil sie der Praxis und dem Denken „einer restlosen Übereinstimmung zwischen den Formen des Staates und dem Zustand der gesellschaftlichen Verhältnisse“ (ebd.) entspricht. Denn die Regierung Cameron will mit den ,big society'-Programm, wie sie selbst erklärt, den Staat benutzen, um die Gesellschaft nach ihrer eigenen Visionen umzugestalten (vgl. Cameron 2009). Mit dem Begriff der Postdemokratie wird also nicht nur eine Tendenz zur detaillierten Erfassung der Gesellschaft, sondern auch eine Festlegung auf die damit einhergehende Zuschreibung einer Position kritisiert. Gezählt werden nur „die wirklichen Teile, die tatsächlichen Gruppen, die durch die Unterschiede in der Geburt bestimmt sind, die Funktionen, Plätze und Interessen, die den Gesellschaftskörper bilden, unter Ausschluss jeden Supplements“ (Rancière 2008: 29). Davon ausgeschlossen bleibt, wer Teil ohne Anteil ist. Dies zeigte sich auch in den Reaktionen des Premierministers auf die riots im August 2011. Die Proteste der Jugendlichen, die sich in den tagelangen Ausschreitungen äußerten, wurden als pure Kriminalität abgetan (vgl. hierzu den Beitrag von Dzudzek/ Müller in dieser Ausgabe).

\section{Are we really all in this together?}

Im Folgenden werden die theoretischen Betrachtungen zur Postdemokratie auf den konkreten Fall der besetzten Gemeindebibliothek bezogen. Auch in Nord-London hat das „One Barnet Transformation Programme”, das sich positiv auf die Gesellschaftsvorstellung der Regierung Cameron bezieht, der lokalen Bevölkerung die Chance genommen, ihr Anliegen - den Erhalt der Bibliothek - zu vertreten, weil es in diesem Rahmen keinen Platz mehr hatte. Eine Mehrheit wählt im Bezirk traditionell konservativ, stand aber nun in einem gewissen Widerspruch zu ,ihrer Partei', die unmittelbar nach der Wahl damit begann, ihre Ideen in die Tat umzusetzen, mit denen sie zuvor geworben hatte. Neben den Prinzipien wie Lokalismus und Transparenz sieht das Konzept der ,big society im Kern drei Punkte vor: die Förderung sozialer Unternehmen, freiwillige Gemeindearbeit und eine Pädagogik der Vorbildwirkung. Dabei wird versucht gesellschaftliche Widersprüche 
$\mathrm{zu}$ neutralisieren indem vor allem Formen von sozialem Engagement gefördert werden, die in marktkonformer Weise organisiert sind. Die Lösung für alle Probleme ist in dieser Politik immer schon gesetzt, es ist der Markt. Ein Beispiel für diesen postdemokratischen Einfluss auf die Politik, liefert die Technik des ,Schubsens', die in der ,big society'-Rede Camerons angesprochen wurde. In dieser fließen Ansätze der Sozialwissenschaften, der Unternehmenspsychologie und der Marktforschung zusammen. Dieses Verfahren des ,Schubsens ' basiert Colin Couch zufolge darauf, Bürger_innen zu einem erwünschten Verhalten zu bewegen, ohne explizite Gesetze oder Verordnungen verabschieden zu müssen. „Das Konzept beruht auf der Idee, Bürger bzw. Konsumenten zu verleiten, bestimmte Dinge zu tun, ohne dass sie dessen gewahr werden - was nichts anderes heißt, als sich ihren Mangel an Wissen oder Information zunutze zu machen" (Crouch 2011: 238). Doch es bleibt nicht nur bei einer Orientierung an der zeitgenössischen Unternehmenskultur. Das ,big society'-Konzept sieht vor, Sozialprogramme und Dienste, die vormals Aufgabe des öffentlichen Sektors waren, auszuschreiben und an private Firmen zu vergeben. Diese Motive finden sich auch im One Barnet Programme wieder, wo sich explizit auf die ,big society bezogen wird (vgl. The Barnet Council 2010). Die Bibliotheksschließung lag also ganz und gar auf Linie der liberal-konservativen Regierungspolitik. Den Bürger_innen war unter diesen Bedingungen nichts geblieben, als die Schließung ihrer Bibliothek hinzunehmen.

Mit der Besetzung und der Wiederaufnahme des Widerstandes musste daher versucht werden, auf verschiedenen Ebenen die Rahmenbedingungen der Auseinandersetzung zu verschieben. Typisch für die Occupy-Bewegung ist, dass sie konventionelle politische Formen der Lobbyarbeit und juristische Auseinandersetzung mit direkten Aktionen verknüpft. Die Besetzung der Friern Barnet Libary war eine Möglichkeit, der Vorstellung der liberal-konservativen Regierung von einer ,big society' eigene Visionen von Gemeinwesen, Zusammenhalt und Solidarität entgegenzusetzen. Die Praxis der Besetzer_innen und Anwohner_innen bestand darin, der zentralen Forderung von Premier David Cameron und auch des konservativen Gemeinderats von Barnet nach einer stärkeren Verantwortung der Community in einer Weise nachzukommen, die nicht vorgesehen und auch nicht erwünscht war. Die Lobrede auf Freiwilligendienste und eine aktive Gemeindearbeit, die - so der berechtigte Vorwurf - lediglich der Legitimierung der staatlichen Kürzungspolitik dienen, wurde durch aktives politisches Handeln der Anwohner_innen herausgefordert. Der Reiz dieser Strategie bestand also darin, es nicht dabei zu belassen, der Regierung eine Verschleierungstaktik und eine Täuschung der Bürger_innen vorzuwerfen, sondern eine Veränderung im Raum vorzunehmen, die das kritisierte Unrecht sinnlich erfahrbar machte.

Es ging in der Friern-Barnet-Libary-Kampagne in zwei verschiedenen Weisen um Legitimität. In der ersten Phase versuchten lokale Aktivist_innen gegen eine Entscheidung von gewählten Politiker_innen und die Praxis der rechtmäßigen Verwaltung zu protestieren. Sie hatten dabei keine wirkliche Chance. Die konservative Regierung war über ein allgemein akzeptiertes Verfahren an die Macht gelangt, die Verwaltung arbeitete bei der Privatisierung der kommunalen Dienstleistungen im Rahmen geltender 
Gesetze. Die Frage der Legitimität schien eindeutig. Am Ende griffen die Bürger_innen zum ersten Mal zu einem Instrument des zivilen Ungehorsams und besetzten für kurze Zeit die Bibliothek. Und obwohl sie mit dieser Taktik vorerst scheiterten, wurde hiermit die Frage der Legitimität in einer neuen Weise gestellt. Dies wiederholte sich, als die Occupy-Aktivist_innen die Proteste neu in Bewegung brachten. Nun wurde polemisch [5] ein Community-Aktivismus gegen die konservative Austeritätspolitik in Stellung gebracht. Der Protest wurde in der Außenkommunikation in Begriffen beschrieben, die formal völlig dem zu entsprechen schienen, was Cameron in seiner Wahlkampfvision als Idealbild entworfen hatte. Die Besetzer_innen und Unterstützer_innen inszenierten sich selbst als aktiven Teil einer von der Regierung viel gepriesenen ,Bürgergesellschaft', die Verantwortung übernimmt und füreinander sorgt. Damit provozierten sie politische und juristische Auseinandersetzungen, in der Lobreden auf die ,big society' unmittelbar mit den Folgen der Einsparung und Privatisierung kommunaler Dienstleistungen konfrontiert wurden. In dem Moment, als die Gerichte und die Polizei darauf vorbereitet wurden, die Besetzung zu beenden, hatten die Besetzer_innen und die Bürger_innen die Diskussion um die Frage der Legitimität der Ratsentscheidung völlig umgedreht (vgl. Patel 2012).

In diesem Sinne geht es bei der Besetzung der Friern Barnet Libary durch die Occupy-Bewegung und den damit verbundenen Aktivitäten nicht mehr nur um den Erhalt einer am Gemeinwohl orientierten kommunalen Einrichtung, sondern um die polemische Subjektivierung einer kritischen Zivilgesellschaft, die offenlegt, dass die Interessen der Anwohner_innen in der offiziellen Politik nichts zählen. Mit der Privatisierung kommunaler Dienstleistungen und damit vormals öffentlicher Räume werden diese zwar zu Kund_innen umgedeutet. In der Realität verhält es sich jedoch so, dass die allgemeine Verfügbarkeit von Dienstleistungen nicht verbessert wird, sondern abnimmt. Während bestimmte Behördendienste, die beispielsweise mit dem Ausfüllen von Formularen verbunden sind, durch Internetplattformen ersetzt werden können, hinterlässt eine aufgrund von mangelnder ökonomischer Effizienz geschlossene Gemeindebibliothek eine erhebliche Lücke vor allem für solche Menschen, die es sich schlicht nicht (mehr) leisten können, Bücher käuflich zu erwerben.

Mit der Einführung von Marktprinzipien für kommunale Infrastrukturen und Dienste der Daseinsfürsorge wird das formale Gleichheitsprinzip verletzt, da der Zugang nun nach den individuellen ökonomischen Ressourcen geregelt ist und einkommensschwache Gruppen zunehmend ausschließt. Wo eine mangelnde Umsetzung des Gleichheitsprinzips im Bereich der öffentlichen Verwaltung im Rahmen institutioneller Beschwerdeverfahren einklagbar sein kann, führt die Ungleichheit auf dem freien Marktes dazu, dass weniger kaufkräftige Kund_innen ein bestimmtes Produkt - in diesem Falle eine Dienstleistung - einfach nicht (mehr) kaufen können. Die vorherrschende Privatisierungspolitik entspricht also nicht nur einer „Herrschaft des Gesetzes zur Reichtumssteigerung“ (Rancière 2011: 70), sie schwächt auch die institutionalisierten Möglichkeiten, gegen die Durchsetzung gesellschaftlicher Ungleichheit vorzugehen.

Daher war es bei dem Kampf um die geschlossene Gemeindebibliothek in Nord-London so wichtig, den öffentlichen Charakter dieses Ortes 
besonders herauszustellen, der dem Gemeinderat und seinem von der Ideologie der ,big society' durchdrungenen Umstrukturierungsprogramm zufolge ein privater Raum sein soll (vgl. Rancière 2011: 69). Dies bedeutet allerdings nicht, die Auseinandersetzung lediglich auf einen Kampf über das richtige Verhältnis von öffentlich und privat zu reduzieren. Vielmehr geht es um „einen Kampf um die Anerkennung derer, die das staatliche Gesetz ins private Leben der minderen Wesen verwiesen hat, als Gleiche und politische Subjekte; und sodann [um] den Kampf um die Anerkennung bestimmter Raumtypen und Beziehungen, die der Verschwiegenheit der Macht des Reichtums überlassen waren, als öffentliche“ (ebd.: 68). Die Besetzung der Friern Barnet Libary konnte mehr als ein Fall normaler Interessensaushandelung zwischen Bürger_innen, Politik und Justiz sein, weil die Occupy-Bewegung mit ihren 99\% eine Trennung des Ganzen von sich selbst vorgenommen hat und auf die Zählung eines Teils ohne Anteil drängte. (vgl. Ranciere 2002: 41)

Die Bevölkerung ist in Bezug auf die Zukunft der Friern Barnet Libary vom Gemeinderat eindeutig übergangen worden. Die große Betroffenheit und Wut über die lokalen Politiker_innen und Behörden, die die Schließung als unausweichlich darstellten, eröffnete ein window of opportunity für soziale Bewegungen, in einem fürs sie eher untypischen Feld aktiv zu werden. Mit ihrem Ansatz der direkten Aktion, mit der sich die OccupyBewegung zumindest in entscheidenden Teilen der Logik der verantwortlichen Verwaltung widersetzte, schufen sie Anknüpfungspunkte nicht nur für lokale Aktivist_innen, sondern auch für andere Teile der Bewohner_ innenschaft. So kam es dazu, dass in einem politisch eher konservativen Bezirk mit einer großen wohlhabenden Mittelschicht ein Dissens zu neoliberaler Kürzungspolitik und zu der Kommerzialisierung öffentlicher Dienstleistungen vertreten werden konnte.

\section{Schwierigkeiten und Herausforderungen in der Praxis der Besetzung}

Es lassen sich, basierend auf den vorangestellten theoretischen Überlegungen und den praktischen empirischen Erfahrungen, drei praktische Herausforderungen der Kampagne zum Erhalt der Bibliothek benennen, die auch über die konkrete Fallstudie hinaus von Bedeutung sind: Erstens gab es rechtliche Probleme zu beachten, zweitens mussten sich die Besetzer_innen intensiv um die Akzeptanz in der unmittelbaren Nachbarschaft bemühen und drittens drohte immer wieder die Gefahr einer Kooptierung des Protests. In juristischer Hinsicht befand man sich in einer Art Grauzone. Die Art und Weise, wie die Besetzung durchgeführt wurde, verstieß nicht direkt gegen bestehende Gesetze, sie war aber auch nicht explizit erlaubt. Im Gegenteil, der Bezirk als Eigentümer des Gebäudes, sah andere Pläne für die Nutzung vor. Hier stießen die Besetzer_innen, die wie die Occupy-Bewegung insgesamt vor allem auf die Macht von direkten Aktionen setzen, auf eine gewisse Schwierigkeit. Auf der einen Seite bieten direkte Aktionen vielfältige Möglichkeiten der Beteiligung. Gerade das Operieren außerhalb eines institutionellen Rahmens eröffnete in Barnet die Chance, den Widerstand gegen die Schließung der Bibliothek wieder aufzunehmen. Zum anderen mussten 
die Besetzer_innen genau darauf achten, den geltenden rechtlichen Rahmen nicht zu weit zu überschreiten. Zum einen wollte man keinen simplen Vorwand für eine polizeiliche Räumung liefern, zum anderen erhoffte man, im Zuge eines Gerichtsverfahrens eine Einigung mit dem Gemeinderat über eine zukünftige Lizenz für den Bibliotheksbetrieb zu erzielen.

Darüber hinaus gab es die Herausforderung, dem eigenen Handeln in den Augen der Anwohner_innen Legitimität zu verleihen. Die Besetzung musste gemeinsam organisiert werden. Dazu mussten zunächst lebensweltliche Gräben zwischen Hausbesetzer_innen und Vorortbewohner_innen überbrückt werden. Für den Erfolg der Besetzung als politisches Projekt war es wichtig, dass die Besetzung in der Nachbarschaft als gerechtfertigte politische Handlung wahrgenommen wird. Bei den teilnehmenden Beobachtungen fiel auf, dass sich die Occupy-Aktivist_innen für bestimmte Gespräche in den nichtöffentlichen Teil der Bibliothek zurückzogen. Diese Trennung von öffentlich und nichtöffentlich machte sich an der ursprünglichen Raumstruktur des Bibliotheksgebäudes fest. Öffentlich war jener Bereich, in dem schon immer die Bücher in den Regalen lagerten, gelesen und ausgeliehen wurden. Nichtöffentlich waren zwei durch eine Tür hinter der Theke betretbare Wirtschaftsräume. Die Nutzung der Raumstruktur war durch die funktionale Koppelung der Besetzung an das politische Projekt Bibliotheksöffnung gebunden. Die Besetzer schliefen, aßen, lasen und lebten zwar in dem Gebäude, sie wohnten aber nicht dort.

Eine weitere Herausforderung beim Kampf um den Erhalt der Bibliothek stellte die Gefahr einer kommunitaristischen Vereinnahmung dar, der insbesondere den kritischen Charakter des bürgerschaftlichen Engagements neutralisiert hätte. Um Erfolg zu haben, musste die Kampagne der Besetzer_innen und Bewohner_innen auf verschiedenen Ebenen wirksam werden. Neben der direkten Aktion der Besetzung musste auch ein Freiwilligendienst organisiert werden. Das heißt, der Bibliotheksservice wurde anteilig von den Occupy-Aktivist_innen und Anwohner_innen übernommen. Ziel der Kampagne war es aber von Anfang an, auch eine vom Bezirk bezahlte Bibliothekarsstelle zu erkämpfen, um nicht einfach eine öffentliche Dienstleistung durch ein freiwilliges und unbezahltes privates Engagement zu ersetzen. Nach langem Kampf wurden letztlich der Erhalt einer Lizenz erstritten, die eine Nutzung der Bibliothek in den nächsten zwei Jahren sichert, sowie die Zusage des Gemeinderats, die geforderte Stelle zu finanzieren.[6]

\section{Theoretische Probleme des Politische bzw. der Demokratie bei Rancière}

Im Folgenden soll auf einige Probleme eingegangen werden, die die Theorie von Rancière, sowie deren Anwendung auf den Fall der Friern Barnet Library betreffen. Oliver Marchart hat auf eine in der Theorie angelegte begriffliche Engführung von Demokratie und Politik hingewiesen. Er wirft Jacques Rancière einen emanzipatorischen Apriorismus vor, der empirisch nicht überzeugt, „,sondern letztlich nur axiomatisch [...] behauptet werden kann, so unplausibel er angesichts der politischen Realität auch erscheinen mag“ (Marchart 2010: 183). Marchart schlussfolgert, dass für Rancière Politik 
immer (!) eine Politik der Gleichheit und damit emanzipatorische Politik ist - oder eben gar keine Politik ist. Er verweist, darauf, dass bereits Ernesto Laclau in seinem Werk On Populist Reason gegenüber Rancière eingewandt hat, dass das Volk als historischer Akteur nicht immer nur automatisch um eine progressive Identität herum konstruiert werden kann (Laclau 2005:246.). Es besteht beispielsweise auch die Möglichkeit, in einer faschistischen Weise einen Antagonismus im Namen des ,Anteils der Anteillosen“ zu artikulieren. Rancière beschreibt also den Prozess einer politischen Subjektivierung durch einen Streit, unterschätzt aber, dass dieser Vorgang auch reaktionäre Momente und Forderungen stärken kann.

Ein weiterer Einwand, der mit dem Vorwurf des emanzipatorischen Apriorismus verknüpft ist, besteht darin, dass Demokratie/Politik nur als eine kurze punktuelle Unterbrechung der Polizei gedacht wird. Rancière schreibt: „Es gibt Politik, wenn die als natürlich vorausgesetzte Logik der Herrschaft durchkreuzt wird. Das bedeutet, dass es nicht immer Politik gibt. Es gibt sie sogar wenig und selten" (2002: 29). So verlockend es sein mag, diese Theorie auf die Subjektivierung der $99 \%$ als Anteil der Anteillosen anzuwenden, entgeht einer allzu wörtlichen Übertragung der Rancière'schen Definition der Demokratie doch, dass für die Occupy-Bewegung nicht nur eine Präsenz im öffentlichen, von ihr wieder politisierten Raum von entscheidender Wichtigkeit war, sondern auch die Dauerhaftigkeit ihrer Proteste. Occupy ist nicht nur ein symbolisch aufgeladener Slogan oder ein zur Aufrechnung eines Unvernehmens geeignetes Bild. Die Besetzung eines Platzes ist darüber hinaus auch ein Prozess und zwar ein in Raum und Zeit erstreckter Prozess.

Rancières Theorie hilft $\mathrm{zu}$ verstehen, wie und wann demokratische Politik mehr sein kann als die innerhalb eines vorgegebenen institutionellen Rahmens stattfindende Aushandlung von Interessen durch anerkannte sowie ins politische System fest integrierte Akteur_innen. Ein solches demokratisches politisches Handeln kann es allerdings weitaus häufiger geben, als Rancière dies theoretisch postuliert. Occupy lässt sich nicht nur als ereignishaft auftretender ,Anteil der Anteillosen', sondern auch als Prozess interpretieren, bei dem es um den langwierigen Aufbau einer kritischen Gegenöffentlichkeit und -kollektivität geht. Dies zeigt sich vor allem, wenn auch die Aktivitäten der Bewegung nach dem Ende der großen Platzbesetzungen betrachtet werden, darunter die Kampagne rund um die Friern Barnet Libary in Nord-London. Die Bibliotheksbesetzung fand zu einem Zeitpunkt statt, als die Politik bereits Strategien entwickelt hatte, um Massenaktionen im öffentlichen Raum einfacher unterdrücken und kriminalisieren zu können. So sorgten geänderte Sicherheitsund Ordnungsgesetzen dafür, dass Platzbesetzungen nicht mehr in einer rechtlichen Grauzone stattfinden, sondern eindeutig illegal sind. Damit verschwand die Occupy-Bewegung allerdings nicht - weder in den USA noch in London. Die Bewegungen standen allerdings vor der Herausforderung, ihre Praxen an die neue Situation anzupassen.

Die Aktivitäten der Occupy-Bewegung waren von Anfang an auf eine gewisse Dauer hin angelegt. Zum einen brachte die Besetzung von und das Campen auf öffentlichen Plätzen symbolisch sehr deutliche eine Differenz zum Ausdruck, weil die Kontrastierung der von Macht strotzenden Glas- und 
Stahlbauten großer Konzerne mit den ärmlich wirkenden Zeltstädten besonders sinnfällig die Spaltung der Gesellschaft vor Augen führt. Zum anderen gab es aber von Beginn an rund um die Zeltstädte in London noch weitere Besetzungen von leerstehenden Räumen, um diese für Bildungs- und Stadtteilaktivitäten zu nutzen. Dies zeigt eine zusätzliche Komponente von Occupy London, die daran besteht, neue Beziehungen zwischen Gruppen und Menschen zu schaffen, die sich zuvor oft völlig unbekannt waren. Diese langwierige Aufbau- und Organisationsarbeit ist mit einer Theorie wie der von Jacques Rancière nicht ohne Weiteres einzufangen. Diesen Aspekt auBer Acht zu lassen, könnte aber bedeuten, sich den Blick auf strategische Optionen für linke Politik in der postpolitischen Stadt zu verbauen.

Obwohl Forderungen wie die nach einer Kontrolle der Banken, der Einführung einer Transaktionssteuer oder dem Erhalt des staatlichen Gesundheitsdienstes sich zwar nicht direkt an eine bestimmte Partei wenden, sind es eindeutig Forderungen, derer sich eine Regierungspartei annehmen könnte. Nicht alle Äußerungen, die im Rahmen der Proteste der Occupy-Bewegung getätigt wurden, standen also in einem radikalen Widerspruch zum bestehenden politischen System. Die alltägliche Praxis der Occupy-Bewegung - das fürsorgliche Miteinander, die Selbstorganisierung von Essen und Sicherheit im Camp, die politische Bildungsarbeit und nicht zuletzt die Aushandlung von Entscheidungen in den Vollversammlungen wirft die Frage auf, ob hier nicht ein Verständnis von politischem Handeln vorherrscht, das den Staat zwar kritisiert, aber keine Anstalten macht, ihn zu verbessern oder die Macht zu erringen. Eine Gefahr könnte dabei für die Protestierenden darin bestehen, die Frage nach den Gelingensbedingungen der Subjektivierung konsequent auszusparen und die Frage von Strategie und Taktik zum Gelingen des eigenen Projektes nicht zu stellen (vgl. Kastner 2012: 62). Über dieses Problem lässt sich theoretisch trefflich streiten. In der hier untersuchten Praxis von Occupy London nach der Räumung der symbolisch wichtigen Platzbesetzungen zeigt sich, dass sehr wohl eine Abwägung von Strategie und Taktik stattfand, die auch klare Kriterien für den Erfolg des eigenen politischen Handelns formulierte. Als notwendig erachtet wurde eine Umorientierung: weg von der sehr breiten und vielstimmigen, aber auch eher abstrakten Kritik an der Austeritätspolitik der Regierung und anderen Auswüchsen des Neoliberalismus mithilfe von großen und öffentlichkeitswirksamen Platzbesetzungen hin zu lokal eingrenzbaren Aktionsfeldern, Kämpfen und konkreten Projekten, verbunden mit einer Strategieplanung, wie diese politisch durchzusetzen und zu gewinnen sind, ohne die Bezugnahme auf die ,großen Themen' wie krisenhafter Kapitalismus und neoliberale Postpolitik aufzugeben.

\section{Das In-Erscheinung-treten des kollektiven Raumes}

Das Unterfangen, die Theorie der Postpolitik bzw. Postdemokratie als eine Art Deutungsfolie für die Analyse einer konkreten lokalen Praxis zu nutzen, ist mit einigen Fallstricken verbunden. Solche Theorien werden politische Handeln in den seltensten Fällen im gleichen Maße anleiten können, wie dies in einem naturwissenschaftlichen Experiment möglich ist. Bei der Untersuchung von konkreten politischen Handlungen und Praxen wird 
man also immer auch auf Aspekte stoßen, die nicht zur theoretischen Folie passen. Trotzdem hat es sich als aufschlussreich erwiesen, die Kampagne der Occupy-Aktivist_innen in Barnet mithilfe von Rancière zu durchdenken. Der Beitrag seines theoretischen Ansatzes zum besseren Verständnis lokaler Kämpfe besteht in der Erklärung, wie eine Situation politisiert werden kann. Dies ist der Fall, wenn ein spezifischer Kampf, „wenn die partikulare Forderung beginnt, als eine metaphorische Verdichtung des umfassenden Widerstandes gegen die da oben, gegen die, die an der Macht sind, zu funktionieren, so dass der Protest in Wirklichkeit nicht länger allein von dieser speziellen Sachfrage handelt, sondern von der allgemeinen Dimension, die in jenem partikularen Anliegen mitschwingt“ (Žižek 2010: 281).

Die Besetzung der Bibliothek in Barnet liefert ein instruktives Beispiel einer solchen demokratischen Politik des Politischen in der postpolitischen/postdemokratischen Stadt. Es illustriert, wie sich lokale Auseinandersetzungen gegenüber einer umfassenderen Analyse und Kritik an neoliberalen Politiken öffnen lassen. Der beschriebene lokale Kampf stellt damit auch eine spannende Übersetzungs- und Verknüpfungsleistung dar. Er ist auch deswegen von Relevanz, da vergleichbare Kürzungsmaßnahmen im ganzen Lande stattfinden bzw. vorgesehen sind. Da das „One Barnet Transformation Programme“ stellvertretend für eine neoliberale Postpolitik betrachtet werden kann (vgl. Conway 2013), ist auch von Bedeutung, wie sich die Gegenwehr formiert hat, welche politischen Allianzen eingegangen wurden und was deren Errungenschaften waren. Wie gezeigt, ist es dem Bündnis aus lokalen Aktivist_innen, Anwohner_innen und der OccupyBewegung in Barnet gelungen, siegreich aus ihrem Kampf hervorzugehen. Relativ eindeutig war im Vorfeld der Kampagne der Erhalt der Bibliothek als konkretes Ziel identifiziert worden, das durch die Besetzung und die begleitenden Aktionen erkämpft werden sollte. Hier liegt der Vorteil gegenüber abstrakteren Protesten beispielsweise gegen die Kultur des Finanzmarktkapitalismus, die schnell zu einer Demobilisierung führen können, weil nicht klar ist, wofür genau man eigentlich kämpft.

Dagegen hat es das Bündnis aus Besetzer_innen und Unterstützer_innen in Barnet verstanden, auf verschiedenen Ebenen zu operieren und die Forderung nach einem Erhalt der Bibliothek und die entsprechende finanzielle Unterstützung durch den Bezirk auf eine recht intelligente Form zu artikulieren und in die Öffentlichkeit zu tragen. Der unmittelbare Erfolg der Kampagne besteht darin, für die Bibliothek eine Lizenz erkämpft zu haben, der ihren Fortbestand für die nächsten zwei Jahre sichert. Die Kampagne für die Wiedereröffnung der Friern Barnet Libary hatte zudem offenbar auch Vorbildcharakter. Nach der Rettung der bezirklichen Bibliothek kam es im Nachbarstadtteil Enfield zu einer ähnlichen Mobilisierung. Aktivist_innen besetzten dort ein vom Gemeinderat geschlossenes Familienzentrum, mit Bezugnahme auf den errungenen Erfolg in Barnet (vgl. Occupy London 2013).

\section{Endnoten}

[1] Die Ausführungen basieren auf teilnehmender Beobachtung und Interviews, die der Autor im Herbst 2012 durchgeführt hat. Eine Woche lang war es ihm möglich, die Bibliothek täglich zu besuchen und an den Aktivitäten der Occupy-Aktivist_innen 
teilzunehmen. Er half beim Sortieren und Einräumen von Büchern, bei der Abholung von Sachspenden aus der Nachbarschaft, beim Einkauf von Lebensmitteln und bei der Vorbereitung von Organisationstreffen. Dieses war eine der Bedingungen, die die Occupy-Aktivist_innen dem Autor im Gegenzug für ihre Bereitschaft, ihm Auskunft zu geben und ihm Zugang zu ihren Strukturen zu gewähren, gestellt hatten.

[2] Emma Dowling (2012) zufolge dient der Bezug auf die Big Society allerdings in Teilen auch ganz schlicht dazu, die Austeritätspolitik im Zuge der anhaltenden Krise von 2007 zu rechtfertigen.

[3] Mit ideologisch ist in diesem Fall dieAuffassung gemeint, dass nach dem Zusammenbruch des Ostblocks die Zeit der großen Erzählungen, insbesondere die Zeit des Marxismus, ein Ende gefunden hätte.

[4] Dies hat zur Voraussetzung, dass es einem Großteil der Bevölkerung gelingt, ihren ökonomischen Status zu halten, was angesichts der rasant steigenden Mieten in London durchaus infrage steht.

[5] Das Wort ,polemisch` wird hier im Sinne der älteren Begriffsbedeutung verstanden als das Bloßstellen oder Überführen eines Gegners im Streit.

[6] Damit gelang es zumindest teilweise, der drohenden Einverleibung der Kampagne im Sinne der konservativen Vision von einer ,big society', die möglichst wenig kosten soll, zu entgehen, auch wenn der Kampf der Bürger_innen um ihre Bibliothek als Bestätigung dieser Vision interpretiert werden kann.

\section{Autor_innen}

Johannes Diesing ist Politikwissenschaftler mit den Forschungsschwerpunkten OccupyBewegung, Postdemokratie, Postmarxismus und radikale Demokratie.

johannes.diesing@uni-rostock.de

\section{Literatur}

Badiou, Alain / Rancière, Jacques (2010): Politik der Wahrheit. Wien: Verlag Turia \& Kant.

Bedorf, Thomas / Röttgers, Kurt (2010): Das Politische und die Politik. Berlin: Suhrkamp Verlag.

Blunden, Mark (2012): 'EasyCouncil.' Barnet offloads services to Capita in $£ 1$ bn deal. http:// www.standard.co.uk/news/london/easycouncil-barnet-offloads-services-to-capita-in1bn-deal-8385804.html (letzter Zugriff am 22.3.2013).

Booth, Robert (2012): Battle of Barnet: activists secure legal victory in fight against library closure. http://www.theguardian.com/society/2012/oct/12/friern-barnet-library-cutsfight (letzter Zugriff am 10.9.2013).

Cameron, David (2009): The Big Society. http://www.conservatives.com/News/ Speeches/2009/11/David_Cameron_The_Big_Society.aspx (letzter Zugriff am 22.1.2013).

Conway, Terry (2013): Get ready for the Barnet Spring. http://www.coalitionofresistance. org.uk/2013/02/get-ready-for-the-barnet-spring (letzter Zugriff am 10.2.2013).

Crouch, Colin (2008): Postdemokratie. Frankfurt a. M.: Suhrkamp Verlag.

Crouch, Colin (2011): Das befremdliche Überleben des Neoliberalismus $\square$ Postdemokratie. Berlin: Suhrkamp Verlag.

Dörfler, Sebastian (2013): Occupy: Von den Plätzen in den Alltag. http://www.blaetter.de/ archiv/jahrgaenge/2013/juni/occupy-von-den-plaetzen-in-den-alltag (letzter Zugriff am 10.9.2013).

Dowling, Emma(2012): Big society: The iron fist in a velvet glove. http://www.newleftproject.org/index.php/site/article_comments/big_society_the_iron_fist_in_a_velvet_ glove (letzter Zugriff am 10. 9.2013).

Kastner, Jens / Lorey, Isabell / Raunig, Gerald / Steyer, Hito / Waibel, Tom (2012): Occupy! Die aktuellen Kämpfe um die Besetzung des Politischen. Wien: Verlag Turia \& Kant. 
Kingsley, Patrick(2011): Are the Occupy London protesters just a bunch of 'hippies'? http:// www.guardian.co.uk/uk/2011/nov/28/occupy-london-boris-johnson (letzter Zugriff am 21.1.2013).

Laclau, Ernesto (2005): On Populist Reason. London: Verso.

Marchart, Oliver (2010): Die Politische Differenz. Zum Denken des Politischen bei Nancy, Lefort, Badiou, Laclau und Agamben. Berlin: Suhrkamp Verlag.

Ministry of Justice (2013): Legal Aid, Sentencing and Punishment of Offenders Act 2012. http://www.legislation.gov.uk/ukpga/2012/10/pdfs/ukpga_20120010_en.pdf (letzter Zugriff am 13.1.2013).

Mörtenböck, Peter / Mooshammer, Helge (2012): Occupy - Räume des Protests. Bielefeld: transcript Verlag.

Occupy London (2013): ARC court case won by former Friern Barnet Library activists. http://occupylondon.org.uk/archives/25631 (letzter Zugriff am 12.3.2013).

Patel, Reema (2012): Flourishing of 'Big Society' in Barnet raises major legal questions for councils. http://www.guardian.co.uk/local-government-network/2012/dec/o7/barnetcouncil-library-legal-action-protest-big-society (letzter Zugriff am 21.1.2013).

Prynn, Jonathan (2012): London homes crisis: Rents to go up beyond families' reach. http://www.standard.co.uk/news/london/london-homes-crisis-rents-to-go-up-beyondfamilies-reach-8220608.html (letzter Zugriff am 21.1.2013).

Rancière, Jacques(2002): Das Unvernehmen. Politik und Philosophie. Frankfurt a. M.: Suhrkamp Verlag.

Rancière, Jacques (2008): Zehn Thesen zur Politik. Berlin/Zürich: Diaphanes Verlag.

Rancière, Jacques (2012): Der Hass der Demokratie. Berlin: August Verlag.

Shamsuddin, Nafeesa(2012): Occupy squatters liberate library, locals overjoyed. http:// occupynewsnetwork.co.uk/breakin-news-reclaimed-public-library-latest-occupylondon-venture (05.01.2013).

The Barnet Council (2010): What is the One Barnet programme? http://www.barnet.gov. uk/info/920056/one_barnet_transformation_programme/904/one_barnet_transformation_programme (letzter Zugriff am 16.2.2013).

The Barnet Council (2010): One Barnet a sustainable community strategy. http://www. barnet.gov.uk/download/downloads/id/1812/one_barnet_a_sustainable_community_ strategy_2010-2020 (letzter Zugriff am 22.1.2013).

Žižek, Slavoj (2010): Die Tücke des Subjekts. Frankfurt a. M.: Suhrkamp Verlag.

\section{Occupy London und the squatting of the Friern Barnet Library}

The paper discusses the struggle for the occupied Friern Barnet Libary in North London. This is an example of a,politics of the political' against the privatisation measures of the governing liberal-conservation coalition in which statements of the programmatic vision were turned against the implementation that it should have ideologically legitimised. At first, the conditions of the occupation will be described, and afterwards the events in fall/winter 2012/13 will be looked at in detail. It will be explained why the struggle for the library gives an instructive example for the understanding of contemporary social struggles in the post-political city. The notion of post-politics and post-democracy is elaborated by Colin Crouch as well as Jacques Rancière. This paper will focus mainly on Rancière but will take Crouch's approach also into account. 
